\title{
Novel SIW Based Interdigital Bandpass Filter with Harmonic Suppression
}

\author{
Sinan Kurudere \\ Meteksan Defence Ind. Inc. \\ Ankara, Turkey 06800 \\ Email: skurudere@meteksan.com
}

\author{
Vakur B. Ertürk \\ Department of \\ Electrical and Electronics Engineering \\ Bilkent University \\ Ankara, Turkey \\ Email: vakur@ee.bilkent.edu.tr
}

\begin{abstract}
A novel configuration for substrate integrated waveguide (SIW) based bandpass filter with interdigital type resonators is presented. In the proposed structure, interdigital type resonators consisting of vias and circular caps at top of them are combined into a bowtie SIW structure. SIW is fed by a tapered microstrip line transition providing low loss and good matching. In order to improve harmonic suppression performance of the filter, the dumbbell shaped defected ground structure (DGS) is etched to the ground side of the microstrip line feeding section. The proposed filter structure is put in a conducting box in order to improve attenuation levels at the band edges and prevent radiation to the outside. The prototype is designed at $9 \mathrm{GHz}$ center frequency with $500 \mathrm{MHz}$ bandwidth, analyzed and fabricated for verification. The measurement results are in good agreement with the simulations, and the filter exhibits more than $30 \mathrm{~dB}$ harmonic suppression.
\end{abstract}

Keywords-SIW filters, harmonic suppression.

\section{INTRODUCTION}

Recent advances in communication and radar systems require compact size and high performance microwave filters. In order to satisfy the demand of microwave applications, interdigital type bandpass filters are one of the most widely preferred filter types due to their relatively simple construction, compact size and high selectivity [1]. Design procedures for interdigital filters are discussed in [1] and [2] for cavity structures. The capacitively loaded resonators used in [3] and [4] can be used in order to build SIW bandpass filters since their performances are similar to the resonators of cavity type combline and interdigital filters. In [5], these resonators have been used for building combline SIW filters. Moreover, harmonic performance of the microwave filters is important, the dumbbell shaped DGS, split ring resonators (SRRs) and complementary split ring resonators (CSRRs) are used in order to suppress the harmonic band of the filter [6], [7].

In this letter, an X-band SIW based bandpass filter with interdigital type resonators is designed, analyzed and fabricated. Building one resonator as described in [3] is the first step of the design, then necessary number of resonators are placed at an appropriate distance to each other with interdigital configuration. SIW section is squeezed from the sidewalls through the center which looks like a bowtie and additional 4 vias ( 2 input, 2 output) are placed at the input and output

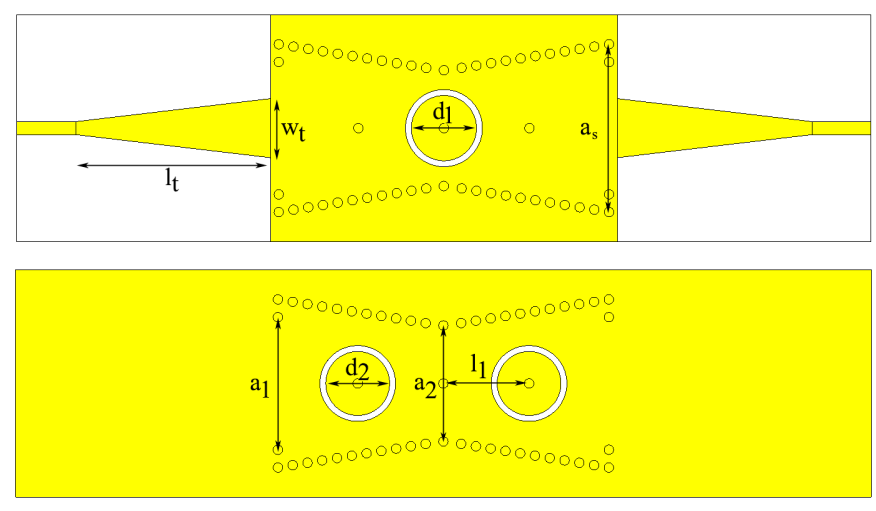

Fig. 1. Configuration of the proposed SIW bandpass filter. Top (upper) and bottom (below) views.

parts of the SIW section to obtain a symmetrical bandpass filter response. A $3^{r d}$ order prototype bandpass filter at $9 \mathrm{GHz}$ center frequency with $500 \mathrm{MHz}$ bandwidth is designed. In order to eliminate the harmonic band of the filter, the dumbbell shaped DGS are etched beneath the microstrip feeding section. A good agreement between simulation and measurement results is achieved and the filter shows high selectivity and good harmonic suppression performance with its compact size.

\section{Filter Design}

Configuration of the proposed filter structure is illustrated in Fig. 1. SIW is designed to operate at X-band and tapered microstrip transition is designed to have good matching at 9 GHz. Inspired from the resonators of [5], resonators are placed in SIW structure with appropriate distance to the adjacent one and the SIW section is squeezed through the center from the sidewalls. Finally, extra metallic vias are added in the input and output of the SIW section.

The design procedure is as follows: firstly, the width $\left(a_{s}\right)$ of the SIW section is determined according to the substrate properties since this width determines the cut-off frequency of the waveguide [8]. The substrate is chosen as Rogers TMM10i with 25 mil thickness since high $\epsilon_{r}=9.8$ will result small size device. Microstrip tapered transition (with parameters $w_{t}$ and $l_{t}$ ) should be used for wideband matching as suggested in [9]. 
After determining the initial SIW dimensions to operate at Xband with microstrip tapered transition, a resonator is placed at the center of the filter. A standard via (drill) dimension is chosen as 16 mil and a gap around the cap is determined by minimum fabrication capability of our facility which is $10 \mathrm{mil}$ for less radiation loss. Cap diameter $\left(d_{1}\right)$ of the resonator is optimized in order to determine the upper cut-off frequency of the filter. Cap with large diameter shifts the upper cut-off frequency to a lower frequency, and vice versa. The diameter is adjusted in order to obtain a notch in $S_{21}$ at about $10 \mathrm{GHz}$ and this will determine the upper cut-off frequency of the bandpass filter. In interdigital filters, the number of resonators determine the filter order, so required number of resonators can be placed at an appropriate distance $\left(l_{1}\right)$ to each other and this distance must also be optimized for the desired center frequency and frequency bandwidth. This is an interdigital type filter, so the resonators are reversed with respect to each other, (i.e., if one resonator's top end is open circuit, then the adjacent resonator's top end will be short circuit) as seen in Fig. 1. Notice that this is a symmetrical filter, dimensions at right and left from the center of the filter are equal. In this study a $3^{\text {rd }}$ order prototype filter is designed, 3 resonators are placed at an appropriate distance to each other. First and last resonators are designed with different diameters $\left(d_{2}\right)$ than the center one (which has diameter $d_{1}$ ) for good matching. The filter response obtained with 3 resonators has a center frequency slightly lower than the actual desired center frequency. It has a broader bandwidth than the desired response and the matching is not satisfactory. The aim of these steps is to obtain a filter response around the desired center frequency and bandwidth. Then, the SIW walls are squeezed from the center wall vias to the center of the filter in order to carry the cut-off frequency of the waveguide to a higher frequency which will affect the lower cut-off frequency of the filter. Bowtie center width of the SIW is described by parameter $a_{2}$ in Fig. 1. Squeezing affects the lower cut-off frequency more than upper cut-off and this modification will result a good matching. Shortly, the resonators affect the upper cut-off and the bowtie width of the waveguide determines the lower cut-off frequency of the filter. The distance between the resonators affects the bandwidth and matching (i.e., larger the seperation, smaller the bandwidth, vice versa). Having observed a filter response, higher frequency attenuation will be better than the lower one. In order to obtain a symmetrical bandpass filter response, additional 4 vias are added to the input and output of the SIW section as depicted in Fig. 1 with distance $a_{1}$. After this last design step, if required, other parameters can be optimized for desired filter response.

Design in this section is made with CST Microwave Studio. $50 \mathrm{ohm}$ microstrip line width is taken as 22 mil, all metallic vias have 16 mil diameter, annular gaps around all resonator caps have 10 mil width. Final design parameters seen in Fig. 1 are given in Table I. Note that the dimensions related to vias are measured from the via centers.

In order to compare this filter with other filters in the literature, we chose SIW based iris filter as a reference filter
TABLE I

DIMENSIONS OF THE SIW INTERDIGITAL FILTER

\begin{tabular}{|c|c|c|c|}
\hline Parameter & Length (mil) & Parameter & Length (mil) \\
\hline$w_{t}$ & 100 & $l_{t}$ & 330 \\
\hline$d_{1}$ & 110.5 & $d_{2}$ & 108 \\
\hline$a_{1}$ & 224 & $a_{2}$ & 196 \\
\hline$a_{s}$ & 284 & $l_{1}$ & 100 \\
\hline
\end{tabular}

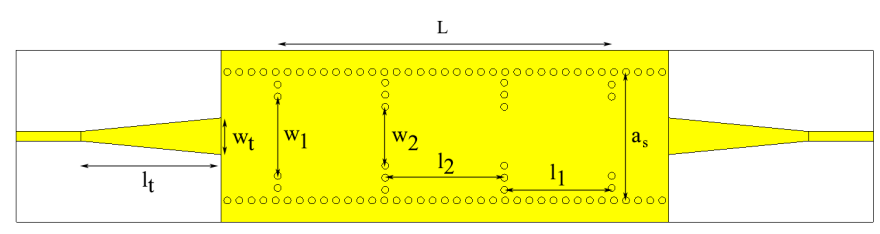

Fig. 2. CST model and parameters of the SIW based $3^{\text {rd }}$ order iris bandpass filter.

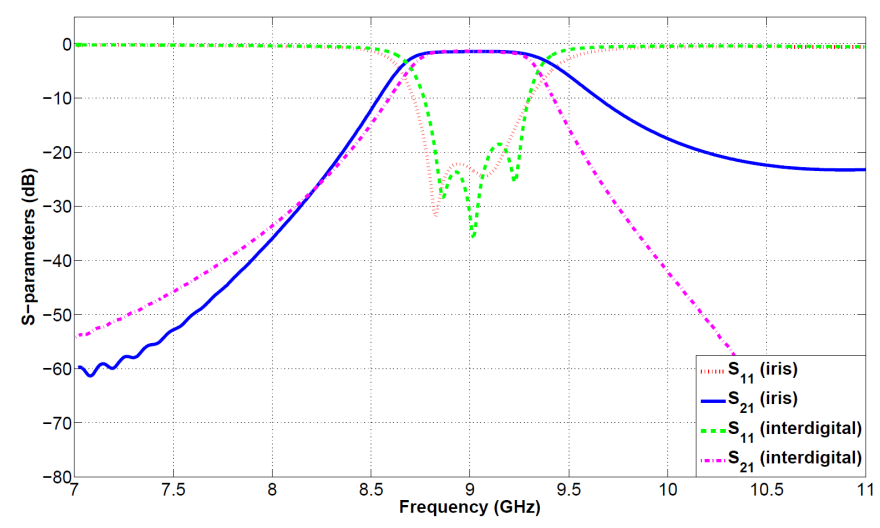

Fig. 3. S-parameter comparison of the proposed filter and iris bandpass filter.

and designed a $3^{\text {rd }}$ order SIW iris filter at the same center frequency with the same bandwidth on the same substrate. Designed filter is illustrated in Fig. 2 and the design parameters (measured from via centers) are given in Table II. This filter is built with 16 mil via diameter and 28 mil via pitch (via to via distance) length. For a fair comparison, only filter sections are measured (Microstrip transition parts are ommitted). Iris filter has 774 mil filter section length (seen as $L$ in Fig. 2). On the other hand, interdigital filter proposed in this work has $560 \mathrm{mil}$ SIW filter length, therefore it is $28 \%$ smaller in length than the iris filter. Besides, when S-parameter results are compared in Fig. 3, proposed interdigital filter has better attenuation at the higher frequency side and both filters almost have the same insertion loss at about $1.3 \mathrm{~dB}$ at $9 \mathrm{GHz}$. Iris filter's out of band suppression is not good because of its topology. For the proposed prototype interdigital filter, the unloaded resonator Q-factor is calculated 290 (including dielectric and conductor losses) at $9 \mathrm{GHz}$.

When harmonic response is investigated, the proposed filter has the harmonic band at about $13.5 \mathrm{GHz}$ which is 1.5 times the center frequency of the filter. Since harmonics are undesired frequency components and must be suppressed, the dumbbell shaped DGS is used at the bottom (ground) side of the microstrip feeding line. In order to investigate 
TABLE II

DIMENSIONS OF THE $3^{\text {rd }}$ ORDER SIW IRIS FILTER

\begin{tabular}{|c|c|c|c|}
\hline Parameter & Length (mil) & Parameter & Length (mil) \\
\hline$w_{t}$ & 85 & $l_{t}$ & 325 \\
\hline$w_{1}$ & 183 & $w_{2}$ & 136 \\
\hline$l_{1}$ & 249 & $l_{2}$ & 276 \\
\hline$a_{s}$ & 297 & & \\
\hline
\end{tabular}

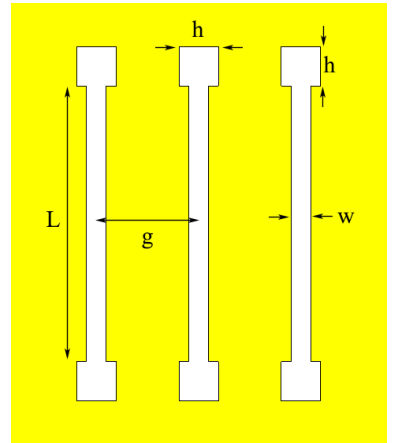

Fig. 4. Parameters of the three dumbbell shaped DGS etched to to ground

the response of the DGS, we designed and simulated one dumbbell shaped DGS underneath the $50 \Omega$ microstrip line. The heads of the dumbbell mostly determine the matching of the structure, and the length of the interconnection between the heads mostly determines the frequency to be suppressed. It behaves like a bandstop filter, and it is tuned in this design in order to suppress the harmonic frequency at around 13.5 GHz. However, one dumbbell shaped DGS is not sufficient to suppress the harmonic of this filter and it does not provide good matching, therefore the number of DGS is increased to 3 which results good suppression at $13.5 \mathrm{GHz}$ and good matching at $9 \mathrm{GHz}$ which is the center frequency of the filter.

A $3^{\text {rd }}$ order DGS is designed as illustrated in Fig. 4 with same dimensions in $1^{\text {st }}$ order case, only the inter-element spacing between each dumbbell is tuned for matching. The dumbbell dimensions shown in Fig. 4 are given in Table III. Top and bottom heads of the dumbbells are square with side length given as $(h)$. The length and the width of the interconnection are given by $L$ and $w$, respectively. The interelement spacing between the dumbbells is given by $g$. Since the function of this structure is to allow transmission of 9 $\mathrm{GHz}$ and reject the harmonic around $13.5 \mathrm{GHz}$, the $3^{\text {rd }}$ order design satisfies the requirements. The designed DGS is etched to the ground side of the microstrip feeding sections which are shown in Fig. 5 for improving the harmonic suppression of the filter. Total length of the filter becomes 1648 mil including the microstrip sections and width is 384 mil. Since dumbbell shaped DGS and annular gaps of interdigital resonators used in this filter are radiating elements, filter is put in a conducting box in order to prevent radiation to outside. Box with $200 \mathrm{mil}$ height is soldered to the top and bottom sides of the filter. Using a conducting box also improves the attenuation at the band edges.

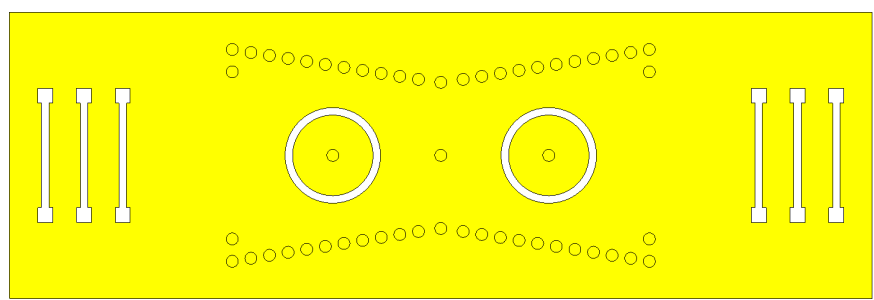

Fig. 5. Dumbbell shaped DGS etched to the ground side of the proposed filter.

TABLE III

DIMENSIONS OF THE DUMBBELL TYPE RESONATORS

\begin{tabular}{|c|c|c|c|}
\hline Parameter & Length (mil) & Parameter & Length (mil) \\
\hline $\mathrm{L}$ & 140 & $\mathrm{~g}$ & 52 \\
\hline $\mathrm{h}$ & 20 & $\mathrm{w}$ & 10 \\
\hline
\end{tabular}

\section{FABRICATION AND MEASUREMENT}

The filter is built on Rogers TMM10i substrate with 25 mil thickness. It has $\epsilon_{r}=9.8$, loss tangent 0.002 (at $10 \mathrm{GHz}$ ) and the conducting material of the filter is copper. As illustrated in Fig. 6, fabricated filters are measured with Southwest Microwave's end-launch SMA connectors due to their easy integration (it does not require soldering) and low loss at 9 GHz. Filters are put in a conducting box, and measurements are made with the box around the filters. Final image of the prototype filter with DGS is given in Fig. 7

For the prototype filter without DGS seen in Fig. 6 (below one), measurement and simulation results are illustrated in Fig. 8. Good agreement in S-parameter (i.e., $s_{21}$ and $s_{11}$ ) results between simulation and measurement is achieved. However, measured insertion loss at $9 \mathrm{GHz}$ is about $2 \mathrm{~dB}$ which is slightly higher than the simulation results. $0.7 \mathrm{~dB}$ difference between simulation and measurement results is caused by the connector losses and unexpected material losses that could not be accounted in the simulations. When the harmonic band of the simulation and measurement is investigated in Fig. 9, the harmonic band occurs around $13.5 \mathrm{GHz}$. In order to suppress the harmonic band, the dumbbell shaped DGS is etched beneath the microstrip feeding section and fabricated prototype is shown in Fig. 6 (upper one). This filter is slightly longer than the previous prototype and has $0.3 \mathrm{~dB}$ more insertion loss both in simulation and measurement. $1.6 \mathrm{~dB}$ loss in simulation and $2.3 \mathrm{~dB}$ loss in measurement results are observed at $9 \mathrm{GHz}$. However the effect of the DGS on harmonic band is shown in Fig. 10. More than $30 \mathrm{~dB}$ harmonic suppression is achieved around $13.5 \mathrm{GHz}$ in measurement results. Notice the radiation around 11 and $12 \mathrm{GHz}$ in Fig. 10. After some EMC analysis, it is concluded that the radiation is caused from the dumbbell shaped DGS underneath the microstrip lines. Since the filter surrounded with a conducting box, DGS radiates into the box.

\section{CONCLUSIONS}

A novel SIW based interdigital bandpass filter configuration with harmonic suppression by the DGS is presented. Capacitive resonators are placed into the bowtie SIW section and the 


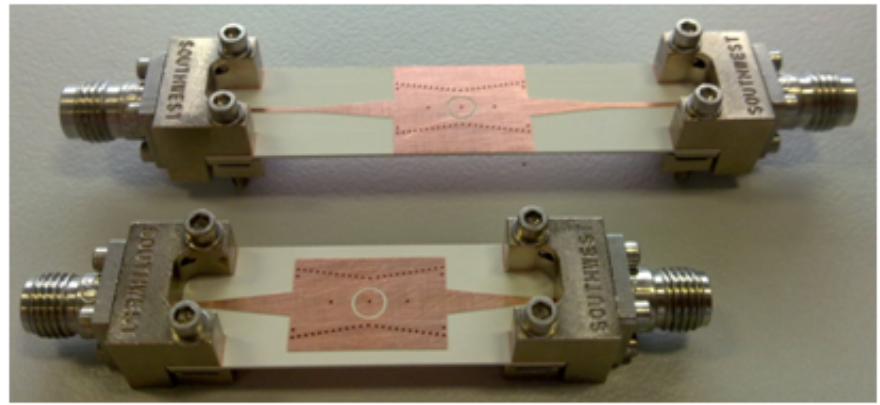

Fig. 6. Fabricated filter images. Upper one is with DGS, below one is without DGS.

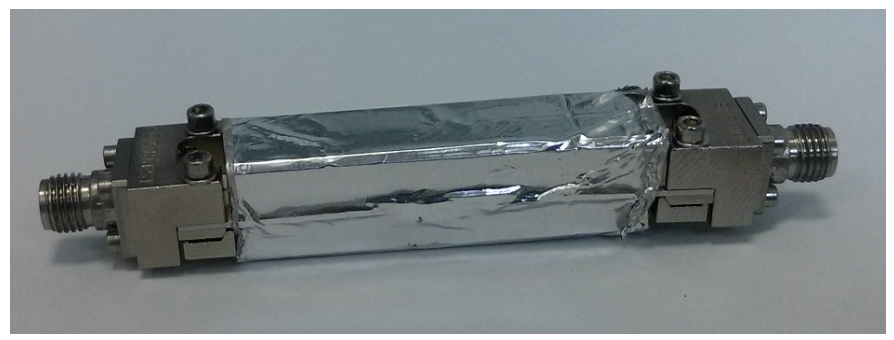

Fig. 7. Fabricated image of the DGS etched filter with a conducting box around it.

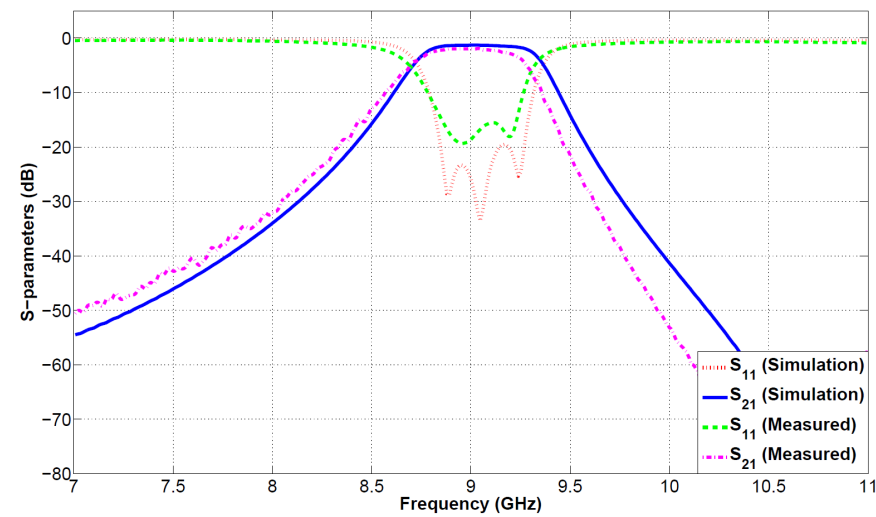

Fig. 8. Simulated and measured S-parameter results of the proposed SIW filter without DGS.

dumbbell shaped DGS provides good harmonic suppression. The filter can be easily built with standard PCB fabrication process and tapered microstrip transition provides easy integration with other printed devices. Besides, high selectivity and smaller size compared to other SIW based filters is the advantage of this filter. Furthermore, very good harmonic suppression in the fabricated filter has been achieved.

\section{REFERENCES}

[1] G. Matthaei, E. M. T. Jones, and L. Young, "Microwave Filters, Impedance-Matching Networks, and Coupling Structures," , Norwood, MA:Artech House, 1980.

[2] R. J. Wenzel, "Synthesis of combline and capacitively loaded interdigital bandpass filters of arbitrary bandwidth," IEEE Trans. Microw. Theory Tech., vol. 19, no. 8, pp. 678-686, Aug. 1971.

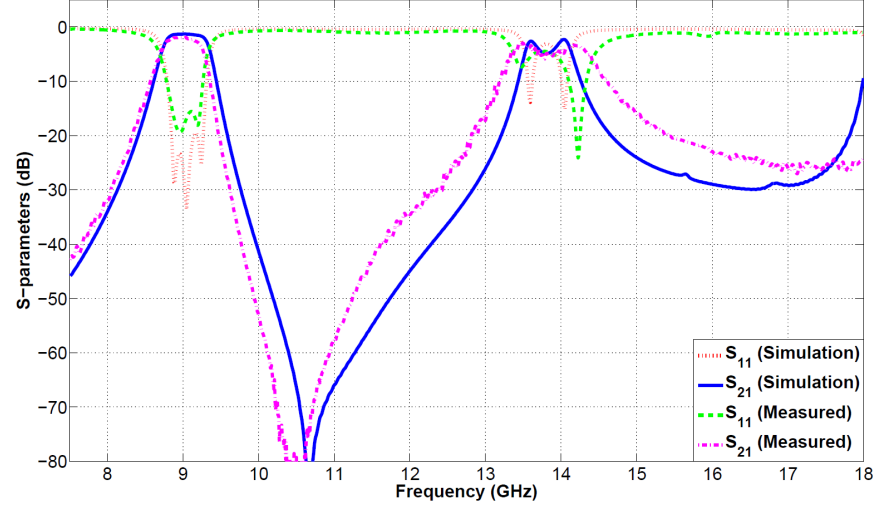

Fig. 9. Harmonic response of the proposed SIW bandpass filter withou DGS.

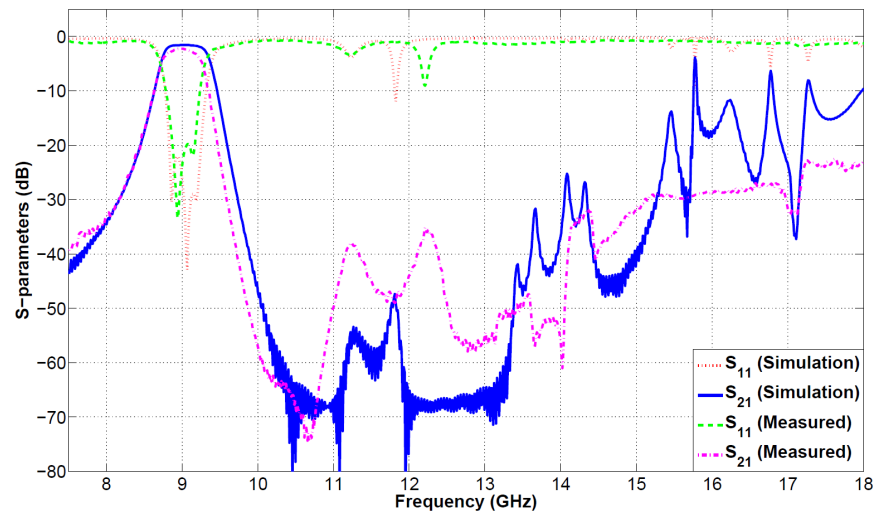

Fig. 10. Harmonic response of the proposed SIW bandpass filter with DGS

[3] J. C. Bohórquez, B. Potelon, C. Person, E. Rius, C. Quendo, G. Tanné and E. Fourn, "Reconfigurable planar SIW cavity resonator and filter," IEEE MTT-S Int. Microwave Symp. Dig., Jun. 2006, pp. 947-950.

[4] J. D. Martinez, M. Taroncher, and V. E. Boria, "Capacitively loaded resonator for compact substrate integrated waveguide filters," in Proc. 40th European Microwave Conference, Paris, France, 2010, pp. 192-195.

[5] J. D. Martinez, S. Sirci, and V. E. Boria, "Compact cpw-fed combline filter in substrate integrated waveguide technology," IEEE Microw. Wireless Compon. Lett., vol. 22, no. 1, pp. 7-9, Jan. 2012.

[6] M. K. Mandal, and S. Sanyal, "A Novel Defected Ground Structure for Planar Circuits," IEEE Microw. Wireless Compon. Lett., vol. 16, no. 2, pp. 93-95, Feb. 2006

[7] F. Falcone, T. Lopetegi, J. D. Baena, R. Marqués, F.Martín and M. Sorolla, "Effective negative- $\epsilon$ stopband microstrip lines based on complementary split ring resonators," IEEE Microw. Wireless Compon. Lett., vol. 14, no. 16, pp. 280-282, Jun. 2004.

[8] M. Bozzi, A. Georgiadis, and K. Wu, "Review of substrate-integrated waveguide circuits and antennas," IET Microw. Antennas Propag., vol. 5, no. 8, pp. 909-920, 2011.

[9] D. Deslandes, "Design equations for tapered microstrip-to-substrate integrated waveguide transitions," IEEE MTT-S Int. Microwave Symp. Dig., May 2010, pp. 704-707. 